\title{
Tris-pyridylmethylamine (TPMA) complexes functionalized with persistent nitronyl nitroxide organic radicals
}

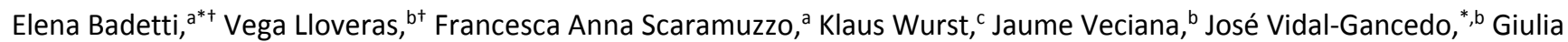 \\ Licini, ${ }^{a}$ and Cristiano Zonta*a
}

\begin{abstract}
The chance to have persistent organic radicals in combination with metals has large interest since it offers the possibility to have new functional molecules with multiple open-shell elements. In this study we report the synthesis of two tripodal tris(2-pyridyl)methylamine ligands (TPMA) functionalized with nitronyl nitroxide persistent radical. The newly formed ligands have been used to coordinate zinc(II), copper(II), iron(II) and cobalt(II). The resulting complexes have been investigated by means of Electron Paramagnetic Resonance (EPR), ESI-MS, FT-IR and X-Ray diffraction. An electron reduction of the N-O radical moiety has been observed, depending on the metal used for the formation of the complex and from the reaction conditions. We have observed small differences in the EPR spectra depending on the meta or para position of the radical moiety in the complex structure and some antiferromagnetic interactions between the paramagnetic $\mathrm{M}(\mathrm{II})$ ions and the radical species.
\end{abstract}

\section{Introduction}

The design of new molecular materials composed by organic nitroxide radicals, ${ }^{1}$ in particular coupled with open-shell metals, ${ }^{2}$ have attracted great attention because of their possible applications in quantum computing, data storage and molecular spintronics. ${ }^{3}$

Many studies have focused on nitronyl nitroxide (NNO) radicals since they are stable under ambient conditions and they are easy to synthesize and functionalize. ${ }^{4}$ In these studies, they often act as bridging ligands for paramagnetic metal ions giving as result the strongest possible magnetic exchange coupling. ${ }^{1}$ This strategy has been used for the synthesis of many magnetic systems such as single-molecule magnets, ${ }^{5}$ single-chain magnets, ${ }^{6}$ and chiral magnets. ${ }^{7}$

While many ligands have been used in combination with NNO radicals, to the best of our knowledge, studies on tris(2pyridylmethyl)amine tripodal ligands (TPMA) have not been reported. ${ }^{8}$

In this context, here we report the synthesis and characterization of a novel family of tris(2-pyridylmethyl)amine tripodal ligands (TPMA) metallic complexes functionalized with NNO radicals. TPMA are a class of ligands that are able to bind a large variety of metal complexes. ${ }^{9}$ In our group, we have also extensively used TPMA in the past years for their application in

\footnotetext{
a. Department of Chemical Sciences, University of Padova via Marzolo 1, 35131 Padova, Italy

b. Institut de Ciència de Materials de Barcelona (ICMAB-CSIC); Campus UAB s/n, 08193 Bellaterra, Barcelona (Spain) and CIBER de Bioingeniería, Biomateriales y Nanomedicina, CIBER-BBN, Barcelona (Spain)

c. Institute of General, Inorganic and Theoretical Chemistry, University of Innsbruck, Innrain 80-82 A-6020 Innsbruck.

+ These authors contributed equally to this work.

Electronic Supplementary Information (ESI) available: [details of any supplementary information available should be included here]. See DOI: 10.1039/x0xx00000x
}

catalysis, ${ }^{10}$ as probes for enantiomeric excess determination ${ }^{11}$ and in the construction of supramolecular cages. ${ }^{12}$ The present study is designed to gather information on: $i$ ) the synthesis two ligands embedding a persistent NNO radical unit in which the metal is not interacting "through resonance" with the organic radical, $i i)$ the effect of the presence of the organic radical on the complexation capabilities of the modified TPMA, iii) some preliminary studies on the interaction among the metal centre and the NNO as function of their distance imposed by the two different ligands. For this purpose, different transition metal TPMA complexes of zinc(II), copper(II), cobalt(II) and iron(II) have been investigated and their magnetic properties have been studied mainly by Electron Paramagnetic Resonance (EPR). The structure of some of these complexes were also elucidated by X-ray analysis. It should be emphasized that the system has been specifically designed to avoid direct the interaction (viz. resonance) of the unpaired electron of the NNO unit with the metal centre, focusing only on the effect of through the space interactions.

\section{Results and discussion}

\section{Synthesis and characterization of the ligands}

Ligands $\mathbf{1} \mathbf{a}, \mathbf{b}$ (Scheme 1), consisting in TPMA core bearing a 3 or 4-formyl-phenyl substituent on one of the three arms was obtained in a two-steps synthesis. The first step was a reductive amination of the commercially available 6-bromo-2pyridinecarboxaldehyde and di(2-picolyl)amine, followed by a Suzuki coupling with 3 or 4-formylphenyl boronic acid respectively. ${ }^{11}$ The NNO derivatives $\mathbf{2 a , b}$ were then synthesized, according to Ullman's procedure, with minor modifications as shown in Scheme 1. In this case, the synthesis started from the commercial 2,3-dimethyl-2,3-dinitrobutane 
which was reduced with zinc in an ammonium chloride buffered solution to yield the key intermediate bis(hydroxylamine). A condensation between the latter and ligands $\mathbf{1} \mathbf{a}, \mathbf{b}$ formed the corresponding dihydroxy-imidazoline intermediates, generally less stable than the corresponding nitroxide derivatives. The dihydroxy-imidazoline intermediate of ligand $\mathbf{1} \mathbf{b}$, with the substituent in position 4 to the pyridine ring, precipitated in the reaction media as a white solid. Once filtered off, it was subsequently oxidized with $\mathrm{NaIO}_{4}$ to give the NNO derivatives $\mathbf{2 b}$ as a blue solid, in good yields. On the contrary, it was not possible to isolate the dihydroxy-imidazoline intermediate of ligand 1a which was directly oxidized to the NNO derivative $\mathbf{2 a}$. The mixture was then purified by precipitation in acetonitrile/diethyl ether, giving the desired compound as a blue solid in $50 \%$ yield. The low yields observed for these reactions were mainly related to the formation of by-products, derived by the electron reduction of the $\mathrm{N}-\mathrm{O}$ moieties. As already known from the literature, this secondary reaction often occurs during the synthesis of NNO systems. The radical ligands $\mathbf{2} \mathbf{a}, \mathbf{b}$ were characterized by ESI-MS, FT-IR and EPR.

FT-IR measurement showed for all the compounds the disappearance of the typical aldehydic carbonyl stretching in the region of $1700 \mathrm{~cm}^{-1}$, the appearance of the $\mathrm{C}=\mathrm{N}$ and $\mathrm{C}-\mathrm{N}$ bond stretching peaks in the region around $\sim 1600$ and $\sim 1400$ $\mathrm{cm}^{-1}$ respectively, and N-O stretching at 1575 and $1372 \mathrm{~cm}^{-1}$. The EPR spectra of free ligands $\mathbf{2 a , b}$ showed at $300 \mathrm{~K}$ in dichloromethane/toluene 1:1 the typical five lines of mono NNO radicals in isotropic conditions with relative intensities 1:2:3:2:1 because of the coupling of the unpaired electron with both the ${ }^{14} \mathrm{~N}$ atoms of the molecule, that resulted equivalent. Their corresponding $g$ values were around 2.0065 and coupling constant with the nitrogens $a_{N}$ around $7.5 \mathrm{G}$ (see Figure S1 and S2). Traces of imino nitroxide impurities, formed after the oxidation of the dihydroxy-imidazoline intermediates, were present in both NNO derivatives $\mathbf{2 a} \mathbf{a} \mathbf{b}$. However, these impurities (lower than $3 \%$ ) detectable by EPR were not appreciable by ESI-MS.

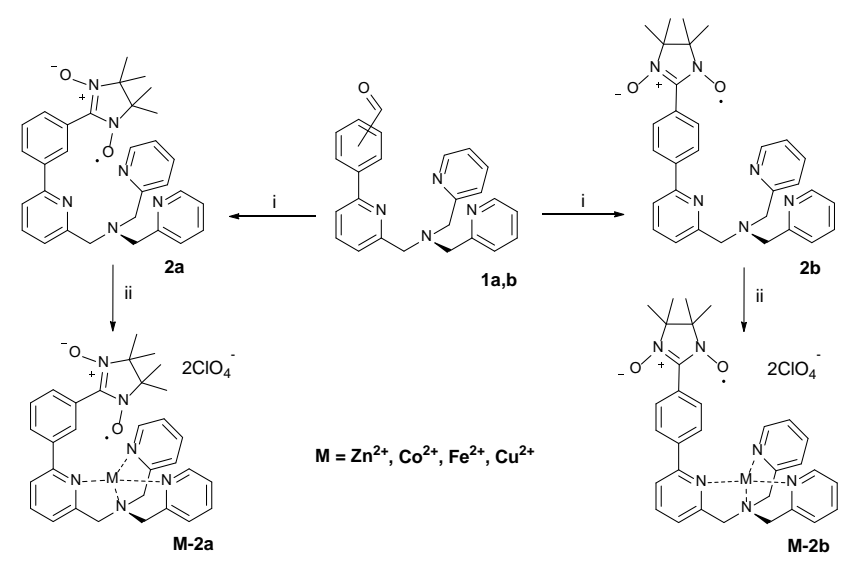

Scheme 1. Synthesis of ligands $\mathbf{2 a}$ and $\mathbf{2} \mathbf{b}$ and corresponding Zinc, Copper and Cobalt(II) M-2a,b complexes. Reaction conditions: i) bis(hydroxylamine), $\mathrm{NaIO}_{4}, \mathrm{MeOH}$; ii) $\mathrm{M}\left(\mathrm{ClO}_{4}\right)_{2}, \mathrm{CH}_{3} \mathrm{CN}$.
As expected, the EPR spectra obtained under frozen conditions $(120 \mathrm{~K})$ showed the typical anisotropic signal of a NNO radical (Figure S3 and S4).

\section{Synthesis and characterization of the $\mathrm{Zn}$ (II) complexes}

The complexes $\mathbf{Z n - 2} \mathbf{a}$ and $\mathbf{Z n - 2} \mathbf{b}$ were obtained by mixing in acetonitrile, at room temperature, ligands $\mathbf{2} \mathbf{a}, \mathbf{b}$ with the commercial zinc(II) perchlorate salt (Scheme 1). Each reaction was let stirring 1 hour at room temperature and purified by precipitation followed by crystallization with diethyl ether. ESIMS experiments, performed in acetonitrile, showed the characteristic isotopic cluster ions for both compounds. The EPR spectra of both $\mathbf{Z n - 2 a , b}$ complexes at $300 \mathrm{~K}$ were similar than the free ligands, but without appreciable imino nitroxide impurities (Figure S5 and S6). In frozen solution they also were similar, showing small differences in the $A_{x}$ and $g_{x}$ between them, obtained from their corresponding simulation (Figure S7 and S8).

\section{Synthesis and characterization of the Co(II) complexes}

Complexes Co-2a and Co-2b were obtained by mixing in acetonitrile, at room temperature, ligands $\mathbf{2} \mathbf{a}, \mathbf{b}$ with the commercial cobalt(II) perchlorate salt and purified by crystallization with diethyl ether. ESI-MS showed the characteristic isotopic cluster ions confirming the formation of the desired compounds. Crystals suitable for X-Ray diffraction were obtained for Co-2b (Figure 1, Table S1). Both Co(II) complexes Co-2a,b displayed similar EPR spectra at $300 \mathrm{~K}$ than the free ligands $\mathbf{2} \mathbf{a}, \mathbf{b}$ (Figure S9 and S10). In frozen solution, we observed the anisotropic spectral pattern of the NNO radical but no clear signal assignable to a cobalt(II) ion was observed in any of them, only an almost unnoticeable signal in each case, which was even less intense in Co-2b compound (see Figure S11 and S12). This fact could be attributed to an antiferromagnetic coupling between the cobalt(II) and the radical ligand. ${ }^{13}$ The temperature dependence of the molar magnetic susceptibility $\chi_{M}$ was measured in the range of 2-300 $\mathrm{K}$ for complex Co-2a (Figure 2) by means of a SQUID magnetometry. A $\chi_{M} T$ value of $2.34 \mathrm{emu} \mathrm{K} \mathrm{mol}^{-1}$ was obtained at $300 \mathrm{~K}$, which is close to the theoretical one expected for two isolated $S=3 / 2\left(\mathrm{Co}^{2+}\right)$ and $S=$ $1 / 2$ (NNO) spins in terms of the spin-only equation $(\chi T=$ $0.12505 \cdot g \cdot 2[S(S+1)]=0.12505 \cdot 2 \cdot 2[3 / 2(3 / 2+1)+1 / 2(1 / 2+1)]=$ 2.25. With decreasing the temperature $\chi_{M} T$ decreased continuously, indicating antiferromagnetic interactions between the spin carriers. 


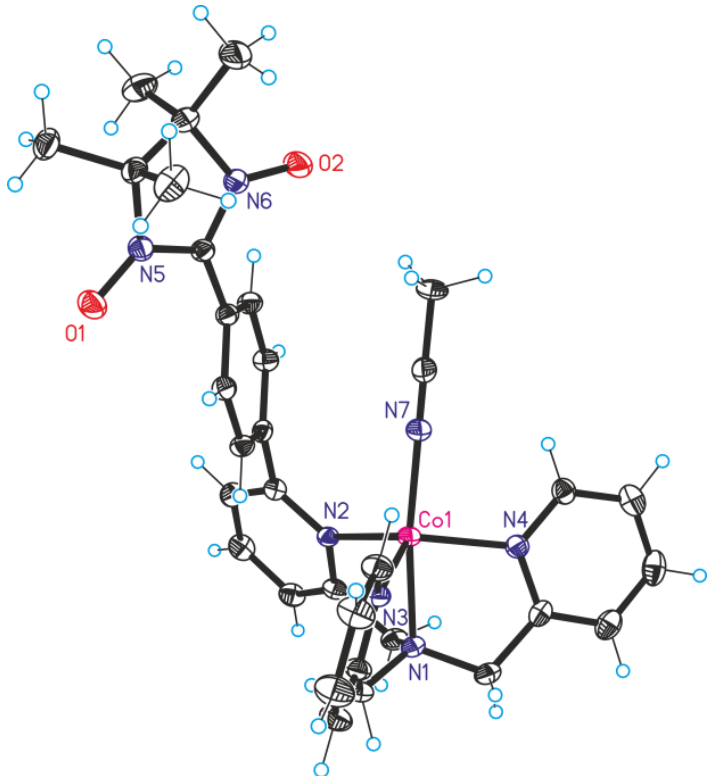

Figure 1. Cationic molecular structure of Co-2b complex, showing thermal ellipsoids a $25 \%$ probability. Selected distances and angles in $\AA$ and ${ }^{\circ}$ : Co-N1 2.163(3), Co-N7 2.042(3), Co-N2 2.095(3), Co-N3 2.039(3), Co-N4 2.065(3), N5-O1 1.285(4), N6-O2 1.283(4) and N1-Co-N7 173.16(12).

Figure 2. Temperature dependence of the molar magnetic susceptibility with temperature $\left(\chi_{M} T\right)$ of complex Co-2a.

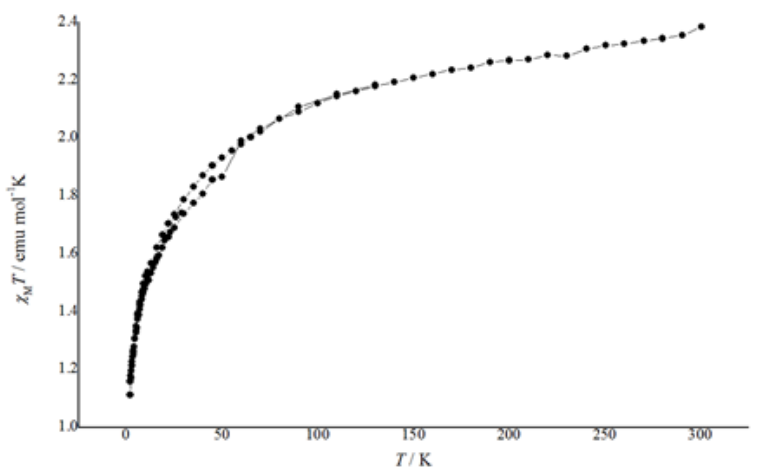

\section{Synthesis and characterization of the Fe(II) complexes}

The synthesis of iron(II) complexes Fe-2a,b was also investigated following the best conditions described for the parent compounds. However, a mixture of two radical species was obtained for the complex synthesized starting from ligand $2 \mathbf{a}$ and iron(II) perchlorate.

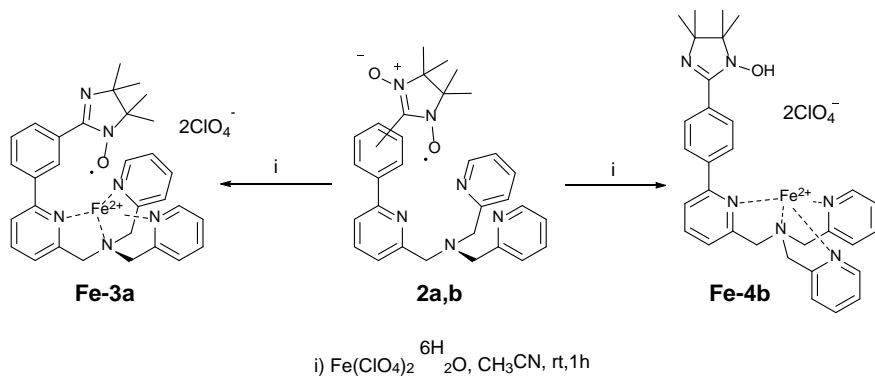

Scheme 2. Synthesis of compounds Fe-3a and Fe-4b.

EPR spectral mixture was obtained, with the principal radical species being the imino nitroxide radical derivatives Fe-3a, and the second one containing 5 lines with $a_{\mathrm{N}(2 \mathrm{~N})}=4.9 \mathrm{G}$ from a radical species with two equivalent $\mathrm{N}$, in a ratio 2.2:1, respectively (Figure S13-S14). ESI-MS $\mathrm{m} / \mathrm{z}$ isotopic cluster ions also indicated that the principal product formed was Fe-3a. In frozen solution, we did not observe any ion metal signal (Figure S15) as it has been shown that Fe(II) complexes coordinated with tris-pyridylmethylamine ligands forms the low-spin complex in acetonitrile, thus, a diamagnetic species. ${ }^{14}$ For the ligand $\mathbf{2} \mathbf{b}$, with the NNO in para position, no radical signal was detected by EPR and ESI-MS analysis, suggesting the formation of the non-radical derivative Fe-4b (Scheme 2). Change of reaction times or stoichiometry did not furnish better results indicating a lower compatibility of the iron(II) with the NNO radical.

\section{Synthesis and characterization of the $\mathrm{Cu}$ (II) complexes}

The procedure previously described for synthesizing zinc(II) and cobalt(II) TPMA complexes was followed to obtain the

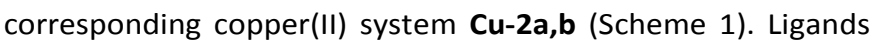
$\mathbf{2} \mathbf{a}, \mathbf{b}$ were mixed with a small excess of the perchlorate metallic salt (less than 1.2 equivalents) to ensure the reaction completeness, and the reaction was let stirring 1 hour at room temperature. However, following these experimental conditions, the EPR spectrum at $300 \mathrm{~K}$ of the copper(II) derivative using ligand 2a showed seven lines with $a_{\mathrm{N} 1}=4.35 \mathrm{G}$ and $a_{\mathrm{N} 2}=9.20 \mathrm{G}$ at $g$-factor $=2.0066$ (Figure 3a), indicating the loss of an oxygen atom from the NNO structure typical of an imino nitroxide radical species, while using ligand $\mathbf{2 b}$ we obtained a cleaner EPR spectrum at $300 \mathrm{~K}$ ( 90\% NNO, and 10\% imino nitroxide), indicating the majority formation of $\mathbf{C u - 2 b}$. XRay diffraction analysis of the former (Figure 4, Table S2) confirmed the loss of an oxygen atom from the structure and showed that the radical species present was an iminium nitroxide (Cu-3a, Scheme 3). The structure showed a change in the coordination geometry from bipyramidal trigonal to octahedral. The EPR spectrum of such a complex in frozen solution (130 K, Figure $3 \mathrm{~b}$ ) also confirmed this geometry. Actually, the spectrum showed hyperfine splitting in the $g_{z}$ region due to coupling of the unpaired electron to the ${ }^{63,65} \mathrm{Cu}(I$ $=3 / 2$ ) nuclei; three of the four metal hyperfine lines were observed and the fourth was partially hidden in the $g_{x, y}$ region. 
a) $300 \mathrm{~K}$
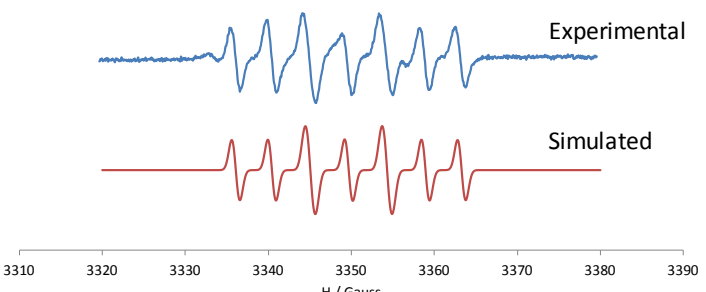

b) $130 \mathrm{~K}$
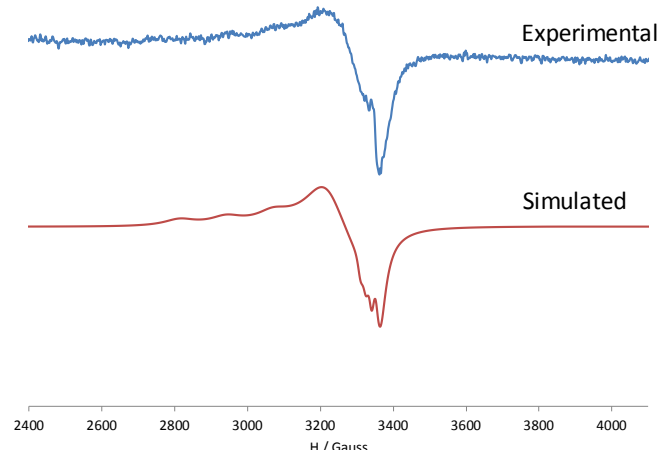

Figure 3. Experimental and simulated EPR spectra of Cu-3a at a) $300 \mathrm{~K}\left(a_{\mathrm{N} 1}=4.35 \mathrm{G}, a_{\mathrm{N} 2}\right.$ $=9.20 \mathrm{G}, g=2.0066$ and $b) 130 \mathrm{~K}\left(\mathrm{~A}_{(\mathrm{x}, \mathrm{x})}=15 \mathrm{G}, \mathrm{A}_{(\mathrm{y}, \mathrm{y})}=5 \mathrm{G}, \mathrm{A}_{(\mathrm{z}, \mathrm{z})}=128 \mathrm{G} ; \mathrm{g}_{\mathrm{x}}=2.020, \mathrm{~g}_{\mathrm{y}}=\right.$ $\left.2.070, g_{2}=2.24\right)$.

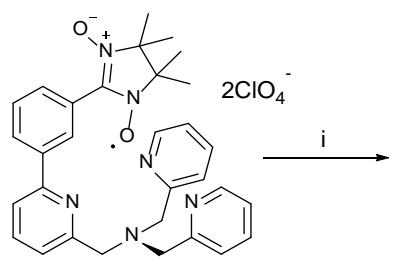

$2 a$

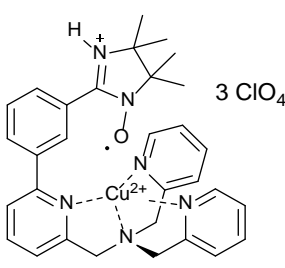

Cu-3a i) $\mathrm{Cu}\left(\mathrm{ClO}_{4}\right)_{2}{ }_{2}^{6 \mathrm{O}}, \mathrm{CH}_{3} \mathrm{CN}, \mathrm{rt}, 1 \mathrm{~h}$

Scheme 3. Formation of compound Cu-3a.

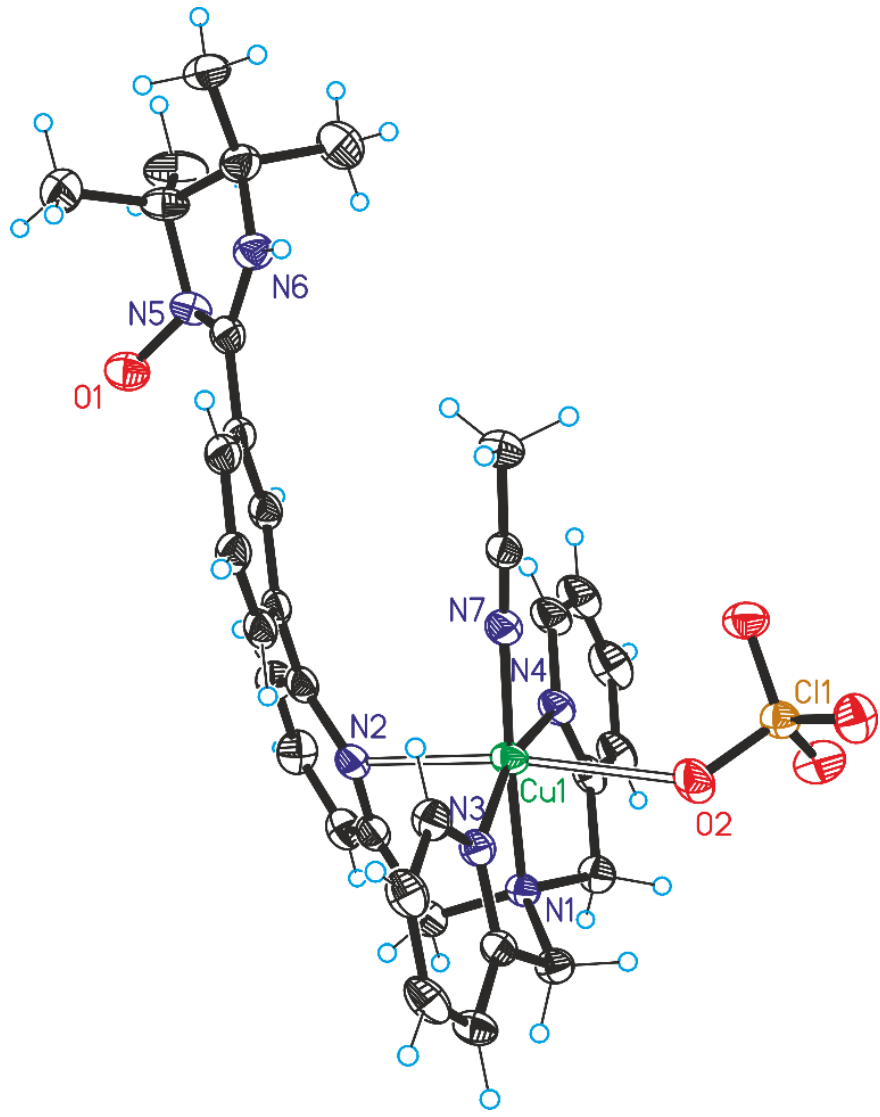

Figure 4. Cationic molecular structure of Cu-3a complex, showing thermal ellipsoids at $25 \%$ probability. Selected distances and angles in $\AA$ and ․: Cu-O2 2.559(4), Cu-N2 2.460(4), Cu-N1 2.021(4), Cu-N7 1.973(5), Cu-N3 1.995(4), Cu-N4 1.993(4), N5-O1 $1.400(7)$ and O2-Cu-N2 166.15(15).

It exhibited a typical $\mathrm{Cu}(\mathrm{II})$ complex possessing a $\mathrm{d}_{\mathrm{x} 2 \mathrm{y} 2}$ ground state $(S=1 / 2)$ with $g_{z}>g_{x} \approx g_{y}>g_{e}=2.0023\left(g_{z}=2.24, g_{y}=2.070\right.$, $\left.g_{x}=2.020\right)$ characteristic of a $\mathrm{Cu}(\mathrm{II})$ complex with an elongated octahedral geometry. ${ }^{15}$

A decrease of the copper(II) perchlorate amount, together with a shorter reaction time, allowed for the synthesis of the desired copper(II) complex Cu-2a (Scheme 1). EPR spectrum analysis confirmed the purity of the compound showing the typical NNO five lines. Both, pure $\mathbf{C u}-\mathbf{2} \mathbf{a}$ and $\mathbf{C u}-\mathbf{2} \mathbf{b}$ complexes showed at $\mathbf{3 0 0}$ $K$ not only the NNO radical signal but also the signal corresponding to a copper(II) ion (Figure 5). In frozen solution, we observed in both complexes the signal of copper(II) ion (Figure 5); in Cu-2a the $g$ parameters obtained from the simulation (see Figure S16) were similar $\left(g_{z}=2.23, g_{y}=2.075, g_{x}\right.$ $=2.032$ ) to the ones from Cu-3a also fulfilling $g_{z}>g_{x} \approx g_{y}>g_{e}$, while in $\mathbf{C u}-\mathbf{2} \mathbf{b}$ they were a little bit different, showing in this case completely axially symmetric $g$-tensor parameters $\left(g_{z}=\right.$ 2.23, $g_{y}=2.075, g_{x}=2.075$ ) fulfilling $g_{z}>g_{x}=g_{y}>g_{e}$ (see Figure S17). The temperature dependence of the molar magnetic susceptibility $\chi_{M}$ was measured in the range of 2-300 $\mathrm{K}$ for complex Cu-2a (Figure 6) by means of a SQUID magnetometry. At room temperature, the value of the $\chi_{M} T$ was $0.70 \mathrm{emu} \mathrm{K} \mathrm{mol}^{-}$ ${ }^{1}$ which corresponds to that calculated for a copper(II) and one NNO radical, i.e. two non-correlated $S=1 / 2$ spins $(0.750$ emu K $\left.\mathrm{mol}^{-1}\right)$. With decreasing the temperature $\chi_{M} T$ decreased 
continuously reaching around 0.350 emu $\mathrm{K} \mathrm{mol}^{-1}$ at $2 \mathrm{~K}$, close to the value of 0.375 emu K mol-1 expected for an isolated unpaired electron. This behavior is indicative of antiferromagnetic interactions between the spin carriers.

Figure 5. EPR spectra in solution at $300 \mathrm{~K}$ and frozen solution at $130 \mathrm{~K}$ of a) $\mathrm{Cu}-\mathbf{2 a}$ and b) Cu-2b.

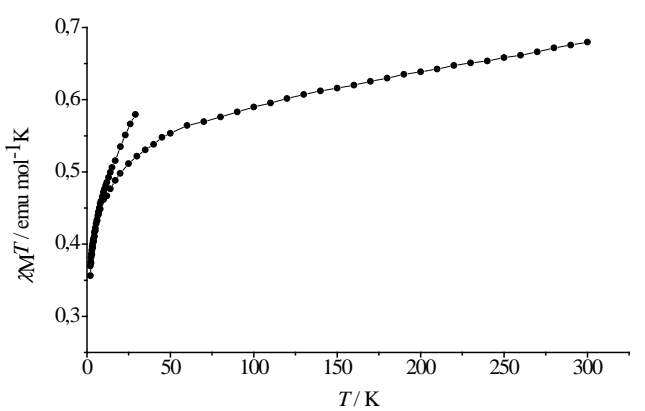

Figure 6. Temperature dependence of the molar magnetic susceptibility with temperature $\left(\chi_{M} T\right)$ of complex $\mathrm{Cu}-\mathbf{2 a}$

\section{Conclusions}

In this paper we have reported the successful synthesis of a new class of tetradentate ligands in which a persistent organic radical is embedded. The ligands have been used to coordinate four different metals and initial studies of their EPR spectra have been recorded. Interesting results have been obtained regarding the radical stability upon complexation. In addition, we have observed some influence of the meta/para position of the radical moiety on their corresponding EPR spectra, as well as some antiferromagnetic interactions between $\mathrm{Co}(\mathrm{II})$ and $\mathrm{Cu}(\mathrm{II})$ paramagnetic metal ions and the organic radical species, which seem more intense in the case of the para substituted complexes. Beside possible interest in the formation of paramagnetic materials, these novel complexes can also find further application in catalysis, where TPMA have already shown outstanding properties.

\section{Conflicts of interest}

There are no conflicts to declare

\section{Acknowledgements}

This work was supported by DGICT-MINECO (MAT2016-80826R) and AGAUR (2017 SGR 918). ICMAB acknowledges Spanish MINECO through the Severo Ochoa Centres of Excellence Programme Grant SEV- 2015-0496.

\section{Notes and references}

¥ CCDC-1997742 Co-2b and CCDC-1997743 Cu-3a contain crystallographic data and can be obtained free of charge from the Cambridge Crystallographic Data Centre via www.ccdc.cam.ac.uk/data_request/cif.

1 I. Ratera and J. Veciana Chem. Soc. Rev. 2012, 41, 303. I. Likhtenshtein. "Nitroxides as Materials". In: Nitroxides. Springer Series in Materials Science, 2020 vol 292. Springer, Cham".

2 A. Caneschi, D. Gatteschi, R. Sessoli and P. Rey, Acc. Chem. Res., 1989, 22, 392. A. Caneschi, D. Gatteschi and P. Rey, Prog. 
Inorg. Chem., 1991, 39, 331. S. Demir, I. R. Jeon, J. R. Long and T. D. Harris, Coord. Chem. Rev., 2015, 289, 149. D. Luneau and P. Rey, Coord. Chem. Rev., 2005, 249, 2591.

3 M.N Leuenberger and D. Loss, Nature 2001, 410, 789. F. Troiani and M. Affronte, Chem. Soc. Rev., 2011, 40, 3119. S. Sanvito, Chem. Soc. Rev., 2011, 40, 3336. M. Castellano, R. Ruiz-García, J. Cano, J. Ferrando-Soria, E. Pardo, F.R. ForteaPérez, S.-E. Stiriba, W.P. Barros, H.O. Stumpf, L. CañadillasDelgado, J. Pasán, C. Ruiz-Pérez, G. de Munno, D. Armentano, Y. Journaux, F. Lloret and M. Julve, Coord. Chem. Rev., 2015, 303, 110 .

4 D. Luneau, Eur. J. Inorg. Chem., 2020, 7, 597-604. V. Lloveras, E. Badetti, J. Veciana and J. Vidal-Gancedo, Nanoscale, 2016 8, 5049. C. Rancurel, H. Heise, F. H. Köhler, U. Schatzschneider, E. Rentschler, J. Vidal-Gancedo, J. Veciana and J. P. Sutter, J. Phys. Chem. A, 2004, 108, 5903. J. VidalGancedo, M. Minguet, D. Luneau, D. B. Amabilino and J. Veciana, J. Phys. Chem. Solids, 2004, 65, 723. M. Minguet, D. B. Amabilino, J. Vidal-Gancedo, K. Wurst and J. Veciana, J. Mater. Chem., 2002, 12, 570. O. Jürgens, J. Vidal-Gancedo, C Rovira, K. Wurst, C. Sporer, B. Bildstein, H. Schottenberger, P. Jaitner and J. Veciana, Inorg. Chem., 1998, 37, 4547.

5 S. Kanegawa, S. Karasawa, M. Maeyama, M. Nakano and N. Koga, J. Am. Chem. Soc., 2008, 130, 3079. J. Wang, J.N. Li, S.L. Zhang, X.H. Zhao, D. Shao and X.Y. Wang, Chem. Commun., 2016, 52, 5033. S. Demir, I.R. Jeon, J.R. Long and T.D. Harris, Coord. Chem. Rev., 2015, 149, 289.

6 A. Caneschi, D. Gatteschi, N. Lalioti, C. Sangregorio, R. Sessoli, G. Venturi, A. Vindigni, A. Rettori, M. G. Pini and M.A. Novak, Angew. Chem., Int. Ed. 2001, 40, 1760. N.R. Liu, Y. Ma, P.P. Yang, X.Y: Song G.F. Xu, J.K. Tang, L.C. Li, D.Z. Liao and S.P. Yan, Dalton Trans., 2010, 39, 3321. M.G.F. Vaz, R.A.A. Cassaro, H Akpinar, J.A. Schlueter, P.M. Lahti and M.A.A. Novak, Chem. Eur. J., 2014, 20, 5460.

7 M. Minguet, D. Luneau, E. Lhotel, V. Villar, C. Paulsen, D.B. Amabilino and J. Veciana, Angew. Chem. Int. Ed., 2002, 41 586. C. Sporer, K. Wurst, D.B. Amabilino, D.R. Ruiz-Molina, H. Kopacka, P. Jaitner and J. Veciana, Chem. Commun., 2002, 20 2342. N. Minguet, D. Luneau, C. Paulsen, E. Lhotel, A. Gorski, J. Waluk, D.B. Amabilino and J. Veciana, Polyhedron, 2003, 22 2349. V. Laukhin, B. Martínez, J. Fontcuberta, D.B. Amabilino, M. Minguet and J. Veciana, J. Phys. Chem. B, 2004, 108, 18441

8 A recent study reports the use of quinonate radicals non covalently bound to a cobalt metal center. G. K. Gransbury, M.-E. Boulon, R. A. Mole, R. W. Gable, B. Moubaraki, K.S Murray, L. Sorace, A. Soncini and C. Boskovic, Chem. Sci., 2019, 10, 8855.

9 T.G. Ribelli, M. Fantin, J.-C. Daran, K.F. Augustine, R. Poli and K. Matyjaszewski, J. Am. Chem. Soc., 2018, 140, 1525. C. Fang, M. Fantin, X. Pan, K. de Fiebre, M.L. Coote, K. Matyjaszewsk and P. Liu, J. Am. Chem. Soc., 2019, 141, 7486. L A. Joyce, M. S. Maynor, J. M. Dragna, G. M. da Cruz, V. M. Lynch, J. W. Canary and E. V. Anslyn, J. Am. Chem. Soc., 2011, 133, 13746. L.A. Joyce, J.W. Canary and E.V. Anslyn, Chem. Eur. J., 2012, 18, 8064. L. You, G. Pescitelli, E.V. Anslyn and L. Di Bari, J. Am. Chem. Soc., 2012, 134, 7117. L. You, J. S. Berman, A. Lucksanawichien and E. V. Anslyn, J. Am. Chem. Soc. 2012, 134, 7126.

10 M. Natali, E. Badetti, E. Deponti, M. Gamberoni, F.A. Scaramuzzo, A. Sartorel and C. Zonta, Dalton Trans., 2016, 45 14764. N. A. Carmo dos Santos, F. Lorandi, E. Badetti, K. Wurst, A.A. Isse, A. Gennaro, G. Licini and C. Zonta, Polymer, 2017, 128, 169. N. A. Carmos do Santos, M. Natali, E. Badetti, K. Wurst, G. Licini and C. Zonta, Dalton Trans., 2017, 46, 16455.

11 F.A. Scaramuzzo, G. Licini and C. Zonta, Chem. Eur. J., 2013, 19, 16809. C. R. Berardozzi, E. Badetti, N.A. Carmo Dos Santos, K. Wurst, G. Licini, G. Pescitelli, C. Zonta and L. Di Bari, Chem.
Commun., 2016, 52, 8428. E. Badetti, K. Wurst, G. Licini and C. Zonta, Chem. Eur. J., 2016, 22, 6515. F. A. Scaramuzzo, E. Badetti, G. Licini and C. Zonta, Eur. J. Org. Chem., 2017, 11, 1438. E. Badetti, N.A.C. Dos Santos, F.A. Scaramuzzo, C. Bravin, K. Wurst, G. Licini and C. Zonta, RSC Advances, 2018 , 8, 19494. N.A. Carmo dos Santos, E. Badetti, G. Licini, S. Abbate, G. Longhi and C. Zonta, Chirality, 2018, 30, 65. F.A. Scaramuzzo, E. Badetti, G. Licini and C. Zonta, Chirality, 2019, 31, 375.

12 C. Bravin, E. Badetti, F. A. Scaramuzzo, G. Licini and C. Zonta, J. Am. Chem. Soc., 2017, 139, 6456. C. Bravin, E. Badetti, R. Putreddy, F. Pan, K. Rissanen, G. Licini and C. Zonta, Chem. Eur. J., 2018, 24, 2936. C. Bravin, A. Guidetti, G. Licini and C. Zonta, Chem. Sci., 2019, 10, 3523. C. Bravin, G. Mason, G. Licini and C. Zonta, J. Am. Chem. Soc., 2019, 141, 11963. C. Bravin, G. Licini, C. A. Hunter and C. Zonta, Chem. Sci., 2019, 10, 1466. C. Bravin, C.A. Hunter, G. Licini and C. Zonta, Chem. Eur. J. 2020 , in press.

13 U. Schatzschneider, T. Weyhermüller and E. Rentschler, Eur. J. Inorg. Chem., 2001, 2569-2586.

14 A. Diebold and K. S. Hagen, Inorg. Chem., 1998, 37, 215.

15 E. Garribba and G. Micera, J. Chem. Educ. 2006, 83, 1229. R. C. Lucacel and I. Ardelean, Int. J. Mod. Phys. B, 2004, 18, 2915. 